\title{
NUCLEAR SPIRALS AS SIGNATURES OF SUPERMASSIVE BLACK HOLES
}

\author{
Hong Bae Ann ${ }^{1}$ and Hyung Mok Lee ${ }^{2}$
}

\begin{abstract}
Recent high resolution images of spiral galaxies show wide varieties of features including nuclear spirals in the central parts. Some of the galaxies show grand-design nuclear spirals. The morphology of grand-design spirals can be further divided by the openness of the arms: tightly wound ones with winding angle of around $3 \pi$ radian and open spirals with winding angle of around $\pi$ radian. Based on hydrodynamical simulations, we have investigated the mechanism responsible for the openness of nuclear spirals. Since the gas flow in the nuclear region is mainly governed by the central mass concentration near the nuclei and the sound speed of the gas, we have examined various models with different mass concentration represented by the mass of the central black hole and different sound speeds. We found that the tightly wound spirals can be formed when the mass of the black hole is large enough to remove the inner-inner Lindblad resonances and sound speeds lie between $15-20 \mathrm{~km} / \mathrm{sec}$. Thus, the presence of the tightly wound nuclear spiral could imply the presence of relatively massive black hole in the center.
\end{abstract}

Subject headings: galaxies: evolution - galaxies: nuclei - galaxies: structure - methods: numerical

\section{Introduction}

Supermassive black holes (SMBHs) are thought to be ubiquitous in galactic nuclei, including the Milky Way (Genzel et al. 2003; Gebhardt et al. 2003). The mass of the SMBH is found to be correlated with the mass of the hot component of the host galaxy, with the largest mass being a few times $10^{9} \mathrm{M}_{\odot}$ (Kormendy \& Richstone 1995; Magorrian et al. 1998). The most direct evidence of the presence of SMBHs at the nuclei of galaxies is the high circular velocities or velocity dispersions of stars and gas in the very vicinity of the galactic centers (Marel \& Roeland 1994; Ferrarese, Ford, and Jaffe 1996). However, most of the kinematical observations are confined to nearby galaxies whose distances are not greater than about 10 Mpc due to the limitation of light gathering power and spatial resolutions available for the present

\footnotetext{
${ }^{1}$ Division of Science Education, Pusan National University, Busan 609-735, Korea

${ }^{2}$ Astronomy Program, School of Earth and Environmental Sciences, Seoul National University, Seoul 151-742, Korea, e-mail: hmlee@astro.snu.ac.kr
}

day ground- and space-based telescopes. This is the reason why the number of galaxies whose SMBHs are identified by spectroscopic observation is quite limited.

Recent high resolution imaging surveys of galaxies show a variety of features such as nuclear rings, nuclear bars, and nuclear spirals in the circum-nuclear regions of normal spiral galaxies (Phillips et al 1996; Elmegreen et al 1998; Laine et al 1999; Carollo et al 2002) as well as active galaxies (Regan \& Mulchaey 1999; Martini \& Pogge 1999; Laine et al 2002). Nuclear bars, sometimes called secondary bars, have attracted special attention in recent surveys of active galaxies (Regan \& Mulchaey 1999; Martini \& Pogge 1999) since they are thought to provide an effective mechanism for fuelling the active galactic nuclei (Shlosman, Begelman \& Frank 1990). But nuclear bars are found to be somewhat rare in active galaxies as well as in normal spiral galaxies compared to the nuclear spirals (Laine et al. 2002; Martini et al. 2003). The high probability $(\sim 50 \%)$ of the presence of nuclear spirals in active galaxies suggests that there is some inter- 
play between the nuclear spirals and the SMBH since the activities of their nuclei are believed to be powered by SMBHs.

The nuclear spirals have a variety of morphology such as grand-design spirals, one-armed spirals, flocculent spirals, and chaotic ones (Martini et al. 2003). The flocculent spirals have multiple arms which are likely to be segmented into smaller arms, while the grand-design spirals have two symmetric arms. The grand-design nuclear spiral is thought to be formed by the hydrodynamic shocks, induced by the non-axis-symmetric potential of the large scale bar (Maciejewski et al. 2002; Ann \& Thakur 2004). Maciejewski et al. (2002) has closely examined the effects of the sound speed on the morphology near the nuclear regions, and concluded that the spiral density waves can penetrate deep into the nuclear region if the sound speed of the gas is sufficiently large ( $20 \mathrm{~km} / \mathrm{sec}$ ). The morphology far from the IILR is nearly independent of the sound speed. In this Letter we further examine the effects of the central potential on the morphology of nuclear spirals in addition to the effects of the sound speed. We are especially interested in how the existence or absence of IILR changes the general morphology in the nuclear region. Since bars do not rotate fast enough to avoid IILR in galaxies with siginificant bulge components, the IILR should be removed by strong mass concentration such as SMBHs.

The morphology of grand-design nuclear spirals can be further divided by the openness of the arms. A good example of the open nuclear spirals is NGC 5427 whose nuclear spiral winds about $\pi$ radian, while that of the tightly wound nuclear spirals is of NGC 5614 whose winding angle is about $3 \pi$ radian (Martini et al. 2003). Figure 1 shows the $H S T$ $H$-band images of these two nuclear spirals. Although more than half of the active galaxies reveal spiral patterns in the nuclear regions, the tightly wound grand-design nuclear spirals are somewhat rare $(\lesssim 10 \%)$.

In this letter, we investigate the physical mechanism that is responsible for the openness of the nuclear spirals using hydrodynamic simulations. Since the gas flow in the nuclear regions is mainly governed by the sound speed of gas and the central mass concentration near the galactic nuclei (Englmaier \& Shlosman 2000), we focus on the effect of the SMBHs and the sound speeds of isothermal

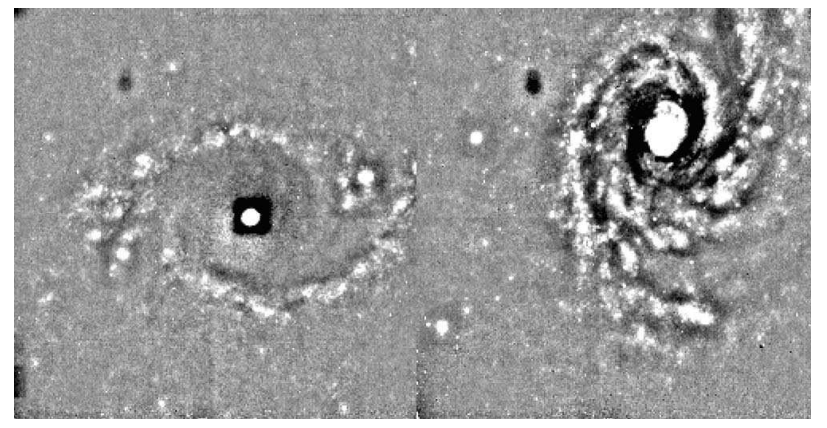

Fig. 1.- Unsharp masked images of two nuclear spirals. The left image is the nuclear spiral of NGC 5427 which is a Seyfert II galaxy with Hubble type of SA(s)c pec. The right image is the nuclear spiral of NGC 5614 whose type is $\mathrm{SA}(\mathrm{r})$ ab pec. Both of the images are taken by $H S T / N I C M O S$ in $H$ band. The size of each image panel is $19 .^{\prime \prime} 2 \times 19 .{ }^{\prime \prime} 2$. The distances of these galaxies are $37 \mathrm{Mpc}$ (NGC 5427 ) and $55 \mathrm{Mpc}$ (NGC 5614). These images are taken from Hubble archives.

gas on the morphology of grand-design nuclear spirals. We describe the models in the next section, and provides the results of simulations in $\S 3$. The implications of the simulations are discussed in the final section.

\section{NUMERICAL MODELS}

We have used the smoothed particle hydrodynamic (SPH) code incorporated with the particle mesh (PM) algorithm for the gravity calculations in order to calculate the response of the gaseous disk to the imposed potential. Our code is basically the same as that of Fux (1999) but the potentials from the building blocks (bulge, disk, halo and bar) are given by analytical functions. We confined our simulations to two-dimensional ones to save computing time and allow for high spatial resolutions within the disk. The disk is assumed to be isothermal gas whose property is determined by the sound speed. The isothermal assumption is clearly ad hoc, but probably a plausible one since the cooling time is much shorter than typical time scales for dynamical processes (e.g., Maciejewski et al. 2002). Since the gas flow depends not only on the imposed potentials but also on the hydrodynamic properties of the ambient gas, we vary the sound speeds of gas as well as the mass models. 
We assumed a logarithmic potential for the dark halo, Plummer potential for the bulge, exponential potential for the disk, and the tri-axial potential for the bar (Long \& Murali 1992), respectively. These potentials are easy to implement and known to reproduce potential of the barred galaxies with suitable choices of parameters (Lee et al. 1999; Ann \& Lee 2000; Ann 2001). We used a point mass potential for the SMBHs with a softening radius of $1 \mathrm{pc}$ to avoid the singularity. The total mass of the galaxy excluding the logarithmic halo is assumed to be $4 \times 10^{10} \mathrm{M}_{\odot}$. The enclosed mass of the halo within $10 \mathrm{kpc}$ is comparable to that of the visible components. In all simulations, we assumed $R_{C R} \approx 1.2 a$ where $R_{C R}$ is the corotation radius and $a$ is semi-axis length of bar. The mass of the bar is taken to be $20 \%$ of the total visible mass and the axial ratio of the bar $(a / b)$ is assumed to be 3 . The mass fractions and scale lengths of the disk and bulge are selected to reproduce the observed rotation curves of typical spiral galaxies. All the potentials are fixed in the frame co-rotating with the bar.

Figure 2 shows the angular frequency curves in the disk for the models with (middle and bottom panels) and without SMBHs (top panel). The global mass distribution for the models of top and middle panels resembles that of late type barred spiral galaxies whose disk-to-bulge ratio $(D / B)$ is assumed to be 5 . The middle panel shows the model with the same mass distribution as that of the model of the top panel but with the mass of SMBH $\left(M_{B H}\right)$ being $0.1 \%$ of the total visible mass of model galaxy, while the bottom panel shows the model for the early type galaxies $(D / B=2)$ with the same $M_{B H}$ as that of the model of the middle panel. As shown in Figure 2, we assumed a slowly rotating bar (pattern speeds of $30.7 \mathrm{~km} / \mathrm{sec} / \mathrm{kpc}$ and $44.8 \mathrm{~km} / \mathrm{sec} / \mathrm{kpc}$ for early type and late type galaxies, respectively) that allows two ILRs which are thought to be necessary for the gas inflow to form nuclear features such as nuclear rings and spirals in the models without SMBH (Ann \& Lee 2000). The corotation radius lies between 3.2 and $3.7 \mathrm{kpc}$, depending on model. The presence of IILR depends on the mass of the SMBHs as well as the global mass distribution of model galaxies.

We have distributed 100,000 particles uniformly within a circular disk of radius $5 \mathrm{kpc}$. Since the resolution length of $\mathrm{SPH}$ is mean inter-particle

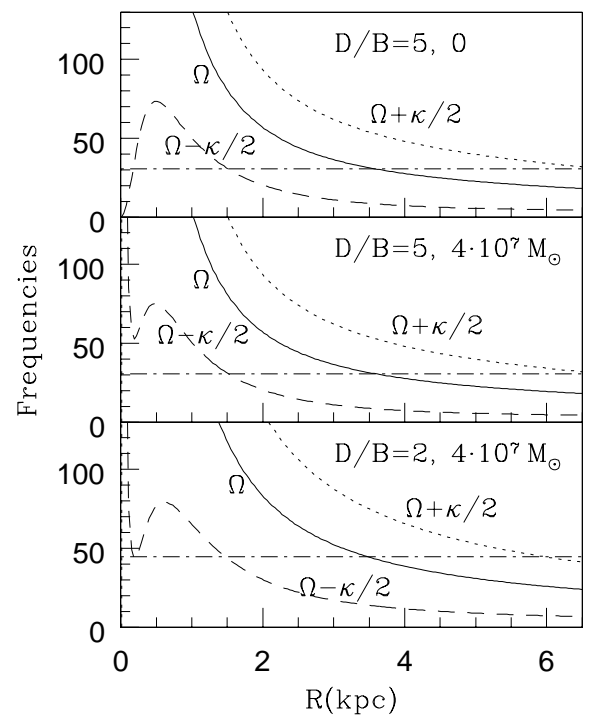

Fig. 2.- Angular frequency curves of three model galaxies. Each model consists of three stellar components (bulge, disk and bar), dark halo and a central black hole. The models differ in $D / B$ ratio and the mass of central black hole, which are given in the upper right part of each panel. The contribution of the bar component is omitted in the plots due to it's non-axisymmetric nature. The horizontal dot-dashed lines represent the pattern speeds of the bars which are selected by the constraint of $R_{C R} \approx 1.2 a$ where $R_{C R}$ is the corotation radius and $a$ is the semi-major axis of bar.

distance, our simulation has initial resolution of around $30 \mathrm{pc}$, which is small enough to study nuclear features in detail. The resolution becomes better than the initial values in the central parts as the density in the nuclear region increases within a few bar rotation periods. Note that the IILR of our model lies around $150 \mathrm{pc}$ from the center.

\section{RESULTS}

We explored a wide range of parameter space for mass models and sound speeds of gas but here we present two types of mass models resembling early and late type barred spiral galaxies. The mass models for early type galaxies are characterized by the disk-to-bulge ratio of $D / B=2$, while those for the late type galaxies are assumed to have the higher disk-to-bulge ratio (i.e., $D / B=$ $5)$. For each type of mass model we vary the sound 


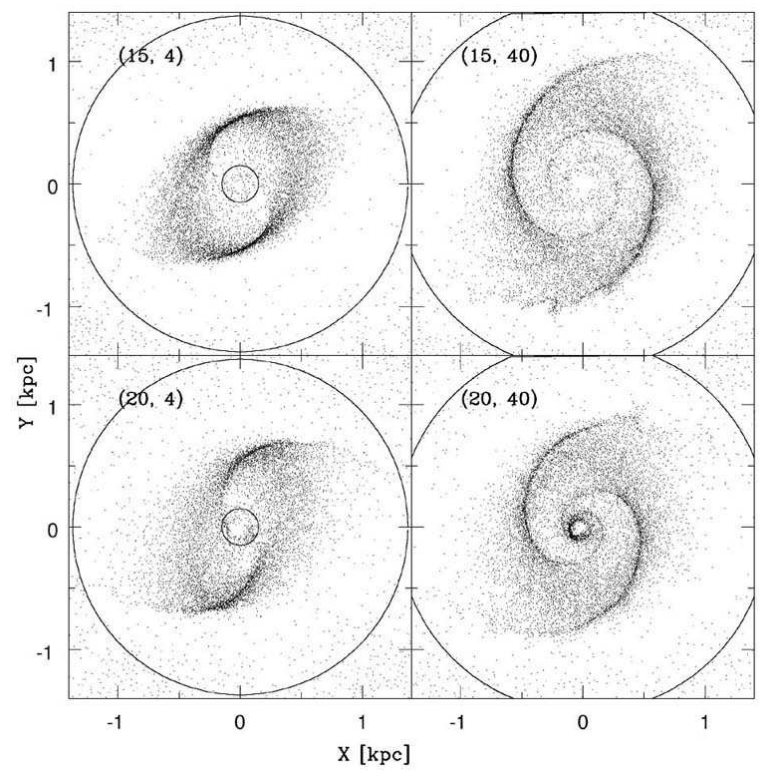

Fig. 3.- Snapshots of the nuclear region of a gaseous disk in the model galaxies that have $D / B=2$ at the evolution time of 5 bar rotations. The models are characterized by the sound speed of gas and the mass of SMBH which are indicated in the upper left corner of each panel. The units of the sound speed and black hole mass are $\mathrm{km} / \mathrm{s}$ and $10^{7} \mathrm{M}_{\odot}$, respectively. The bar always lies horizontally in these figures. The outer circles indicate the location of ILR while the inner circles of left panels indicate the IILR.

speed of gas $\left(c_{s}\right)$ from 5 to $35 \mathrm{~km} / \mathrm{s}$ and $M_{B H}$ from 0 to $1 \%$ of the total visible mass. We found that $c_{s}=15 \pm 5 \mathrm{~km} / \mathrm{s}$ is most plausible for the gas flow that leads to the formation of grand-design nuclear spirals. When $M_{B H}$ is larger than $\sim 0.5 \%$ of the total visible mass, the IILR is likely to disappear.

The nuclear features of gaseous disks of the models for the early type galaxies are shown in Figure 3. They are the snapshots at 5 bar rotations ( $\sim 0.7 \mathrm{Gyr})$, after which the evolution becomes very slow. The models have the same mass distributions except for $M_{B H}$ and $c_{s}$. Thus, the models are characterized by the combination of the sound speed of gas and the black hole mass, which are indicated by the pair of numbers in the upper left corner of each panel in units of $\mathrm{km} / \mathrm{s}$ and $10^{7} \mathrm{M}_{\odot}$, respectively. All the models in Figure 3 clearly show spiral features of two symmetric

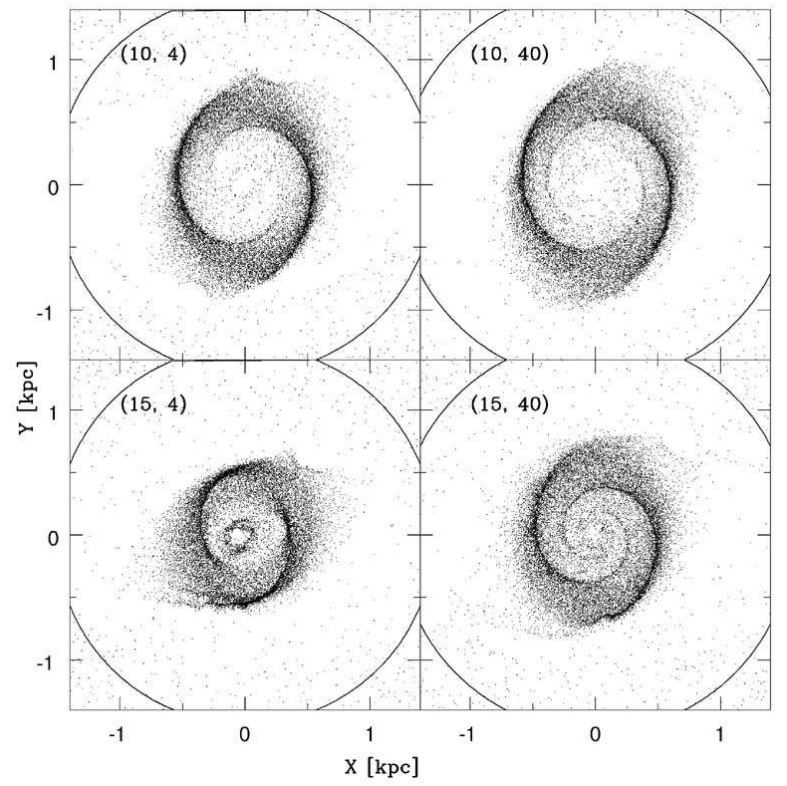

Fig. 4.- Snapshots of the nuclear regions of gaseous disks of models that represents late type galaxies $(D / B=5)$. The evolution time and meaning of the pair of the numbers in the parenthesis are the same as those in Fig. 3. The circles indicate the location of ILR.

arms whose openness depends mainly on the mass of SMBH. The left panels of Figure 3 resemble the open nuclear spiral in NGC 5427, while those of the right panels show more tightly wound spiral features. There are also some differences in the detailed morphology of the nuclear features in the same mass models with different sound speeds of gas. The models with higher sound speeds of gas induce more gas inflow close to the centers. Among the four models, the model with $M_{B H}$ $\sim 1 \%$ of the total visible mass and $c_{s}=15 \mathrm{~km} / \mathrm{s}$ gives the most tightly wound spiral features somewhat similar to the nuclear spiral of NGC 5614.

The evolution of gaseous disks in the models for late type galaxies is somewhat different from that for the early type galaxies. Figure 4 shows the snapshots of the evolution of gaseous disks in the models with $D / B=5$. The masses of SMBHs are the same as those of the early type models but the sound speeds of gas are assumed to be $10 \mathrm{~km} / \mathrm{s}$ and $15 \mathrm{~km} / \mathrm{s}$. There seems to be no nuclear feature similar to the open nuclear spiral of NGC 
5427. Rather, the nuclear features in the models with $c_{S}=10 \mathrm{~km} / \mathrm{s}$, shown in the upper panels of Figure 4, show ring-like nuclear spirals similar to the nuclear ring/spiral of NGC 4314 (Benedict et al. 1998, 2002; Ann 2001) while the model with $M_{B H}$ being $1 \%$ of the visible mass and $c_{s}=15$ $\mathrm{km} / \mathrm{s}$ (lower right panel) shows a tightly wound spiral that reaches close to the center. Similar nuclear features are developed in the simulations that assume the same sound speed of gas and mass model but different $M_{B H}$. Thus, sound speed of $10 \mathrm{~km} / \mathrm{s}$ seems to be not high enough for the hydrodynamic shocks to penetrate deep inside the ILRs to make nuclear spirals there regardless of the degree of the central mass concentration. For higher sound speed of gas $\left(c_{S}=15 \mathrm{~km} / \mathrm{s}\right)$, the models produce tightly wound spiral features when there is an SMBH which is massive enough to remove the IILR.

\section{DISCUSSION}

Non-axismmetric potential by an elongated bar causes substantial non-circular motions of stars and gas, and generates the travelling density wave in disk galaxies. Density wave in the gaseous disk can penetrate the ILR through the hydrodynamic shock since the orbital speed is always much larger than the sound speed in the disk. The loss of orbital energy by the shock causes inflow of the gas. Our high resolution numerical simulations of gaseous disks revealed that the morphology of the nuclear spirals depends strongly on the presence of IILR which usually exists if there is not much concentration of the mass in the center. The IILR can be removed by the SMBH.

There seem to be narrow ranges for the $M_{B H}$ and the sound speed of gas for the formation of tightly wound grand-design nuclear spirals induced by non-axisymmetric potentials. The primary factor that constrains the physical conditions for the formation of tightly wound nuclear spirals is the central mass concentration imposed by $\mathrm{SMBH}$ since the gas flow in the models with relatively low $M_{B H}$ does not lead to the formation of such nuclear spirals. The sound speeds of gas do affect the gas flow but the range of sound speeds plausible for the formation of tightly wound nuclear spirals is limited by the correlation between the gravitational potential shapes and the sound speeds in the nuclear regions of galaxies (Englmaier \& Shlosman 2000). Our simulations show clearly that the plausible gas sound speeds to form the tightly wound nuclear spirals are $15 \pm 5$ $\mathrm{km} / \mathrm{s}$ for late type galaxies and $20 \pm 5 \mathrm{~km} / \mathrm{s}$ for early type galaxies, respectively.

The openness of the nuclear spirals are also known to depend on two parameters, the central mass concentration of the host galaxies and the sound speeds in the gas. However, as shown in Figure 3, the openness of the nuclear spiral depends only on the central mass concentration due to $\mathrm{SMBH}$ for galaxies with small $D / B$. The gas flow in the models with large $D / B$ does not lead to the formation of open nuclear spirals similar to that of NGC 5427. Rather, it leads to the formation of tightly wound but ring-like nuclear spirals when the sound speed of gas is $10 \mathrm{~km} / \mathrm{s}$. The tightly wound nuclear spirals with large winding angles are developed in models with higher sound speed of gas $\left(c_{s} \gtrsim 15 \mathrm{~km} / \mathrm{sec}\right)$. Thus, the openness of the grand-design nuclear spirals may indicate the mass of SMBH that induce the hydrodynamic instability inside the IILR in early type galaxies, while winding angles of the tightly wound nuclear spirals in late type galaxies indicate the degree of the turbulent motion, represented by $c_{s}$ in our models, in the interstellar medium near the galactic nuclei.

The low frequency of tightly wound granddesign nuclear spirals in active galaxies suggests that only a small fraction of active galaxies have SMBHs massive enough to remove the IILR. The minimum mass of SMBH for the formation of tightly wound nuclear spirals depends on the mass of the bulge component whose central concentration dominates the strength of the ILRs. An SMBH having $0.1 \%$ of the total visible mass is massive enough to form tightly wound nuclear spirals in late type galaxies, whereas the minimum mass of SMBH to allow the formation of tightly wound nuclear spirals in early type galaxies is about $0.5 \%$ of the total visible mass of galaxies. This does not mean that tightly wound granddesign spirals are more frequent in late type galaxies than early type ones because the SMBHs in late type galaxies are likely to be smaller than those in the early type galaxies (Magorrian et al. 1998; Ferrarese \& Merritt 2000; Gebhardt et al. 2000).

Therefore, it seems promising that the morphol- 
ogy of the nuclear spirals can be used as a diagnostic for the central mass concentration as well as the kinematic properties of the interstellar medium when it is incorporated with the global morphology of galaxies. More specifically, tightly wound, grand-design nuclear spirals with large winding angle indicate the presence of an $\mathrm{SMBH}$ which is massive enough to remove the IILR.

Our results are based on high resolution SPH simulations, which clearly have some limitations. First, our simulations are purely two-dimensional, which cannot take into account the effects of the vertical structure of the disk. Since the thickness of the gaseous disk is of order of a few hundreds of $\mathrm{pc}$, the vertical structure may not be important for the formation and evolution of the spiral density waves, but this issue can only be studied in full 3dimensional simulations. In order to keep the current resolution, we need to have at least an order of magnitude larger number of particles. Second, the $\mathrm{SPH}$ is known to have significant numerical dissipation. This does not seem to be a serious problem for us since we are assuming isothermal equation of state which itself is a consequence of efficient physical dissipation. The numerical dissipation depends on the resolution of the simulations, but we found very little dependence of the morphology with different number of particles.

We thank H. Kang for valuable discussion and helps in the numerical simulations. This work is supported in part by the grant from ARCSEC (to HBA), and in part by the KRF grant No. 2002041-C20123 (to HML). Most of the computations are conducted using the super computer facilities in KISTI.

\section{REFERENCES}

Ann, H. B., 2001, AJ, 121, 2515

Ann, H.B. \& Lee, H. M., 2000, J. Korean Ast. Soc., 33,1

Ann, H. B. \& P. Thakur, P. 2004, ApJ, Submitted

Benedict, G. F., Howell, D. A., Jorgensen, I., Kenney, J. D. P., \& Smith, B. J., 2002, AJ, 123, 1411

Carollo, C. M., Stiavalli, M., Seigar, M., de Zeeuw, P. T., \& Dejonghe, H., 2002, AJ, 123, 159

Elmegreen,D. M., Chromey, F. R., \& Warren, A. R., 1998, AJ, 116, 2834
Englmaier, P., \& Gerhard, O,1997, MNRAS, 287, 57

Englmaier, P. \& Shlosman, I. 2000, ApJ, 528, 677

Ferrarese, L., Ford, H. C., \& Jaffe, W., 1996, ApJ, 470,444

Ferrarese, L., \& Merritt, D. 2000, ApJ, 539, L9

Fux, R, 1999, A\&A, 345, 787

Gebhardt, K. et al. 2000, ApJ, 539, L13

Gebhardt, K. et al. 2003, ApJ, 583, 92

Genzel, R., 2003, ApJ, 594, 812

Kormendy, J. \& Richstone, D. 1995, ARA\&A, 33, 581

Laine, S. etal. 1999, MNRAS, 302, L33

Lee, C, W., Lee, H. M., Ann, H. B., \& Kwon, K. H., 1999, ApJ, 513, 242

Long, K., \& Murali, C. 1992, ApJ, 397, 44

Maciejewski, W., Teuben, P. J., Sparke, L. S., \& Stone, J. M., 2002, MNRAS, 329, 302

Magorrian, J. et al. 1998, AJ, 115, 2285

Martini, P., \& Pogge, R. W. 1999, AJ, 118, 2646

Martini, P., Regan, M. W., Mulchaey, J. S., \& Pogge, R. W. 2003, ApJS, 146, 353

Phillips, A. C., Illingworth, G. D., MacKenty, J. W., \& Franx, M., l 1996, AJ, 111, 1566

Pogge, R. W., \& Martini, P. 2002, ApJ, 569, 624

Regan, M. W., \& Mulchaey, J. 1999, AJ, 117, 2676

Shlosman, I., Begelman, M. C., \& Frank, J., 1990, Nature, 345, 679

This 2-column preprint was prepared with the AAS LATEX macros v5.2. 